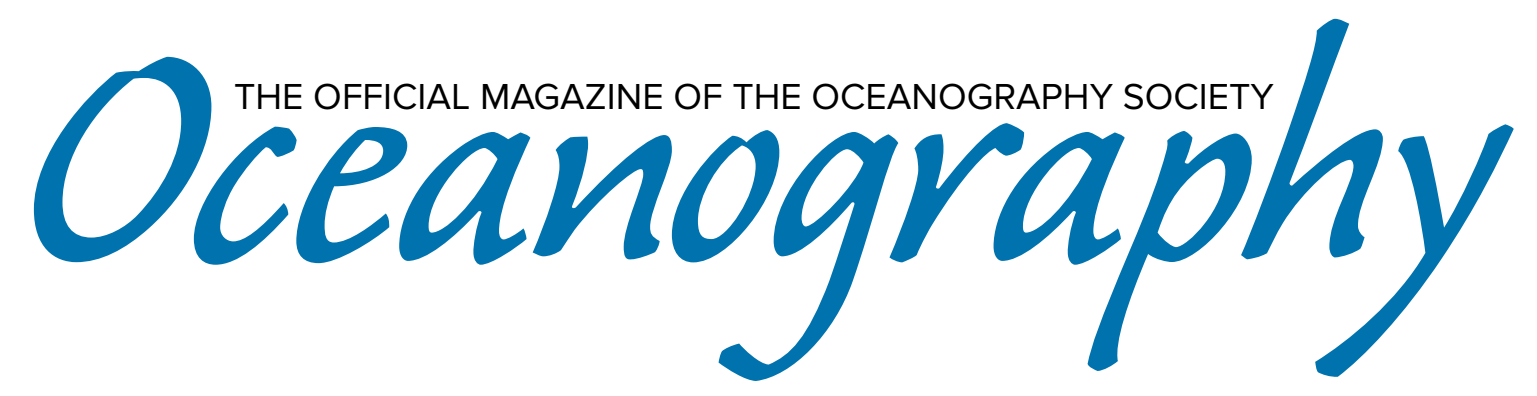

CITATION

Henry, D. 2018. Nonlinear features of equatorial ocean flows.

Oceanography 31(3):22-27, https://doi.org/10.5670/oceanog.2018.305.

$\mathrm{DOI}$

https://doi.org/10.5670/oceanog.2018.305

COPYRIGHT

This article has been published in Oceanography, Volume 31, Number 3, a quarterly journal of The Oceanography Society. Copyright 2018 by The Oceanography Society. All rights reserved.

USAGE

Permission is granted to copy this article for use in teaching and research. Republication, systematic reproduction, or collective redistribution of any portion of this article by photocopy machine, reposting, or other means is permitted only with the approval of The Oceanography Society. Send all correspondence to: info@tos.org or The Oceanography Society, 1 Research Court, Suite 450, Rockville, MD 20850, USA. 


\section{Nonlinear Features of Equatorial Ocean Flows}

By David Henry

ABSTRACT. We examine whether certain oceanographic features of equatorial flows can be modeled using a linear theoretical framework. In particular, we show, using elementary mathematical considerations, that linearization fails to capture the emergence and persistence of large coherent structures that are representative of upwelling and downwelling processes.

\section{INTRODUCTION}

The importance of improving our understanding of ocean dynamics in the equatorial region is well recognized, and has been the subject of much study in recent decades from a variety of different fluid dynamics perspectives (Gill, 1982; McCreary, 1985; Fedorov and Brown, 2009; Constantin and Johnson, 2015; Vallis, 2017; Boyd. 2018). Advancements in ocean data gathering, numerical data analysis, and numerical simulations have furthered our knowledge and awareness of these flows, yet the ability to attain a greater understanding of the fluid motion hinges strongly on achieving progress in the field of theoretical modeling. In fluid dynamics there is often a symbiotic relationship between research of a theoretical nature and that of an observational/experimental nature. Such a correspondence is particularly desirable, and pertinent, in the context of geophysical fluid dynamics (GFD) - by which we mean that the scale of the motion is sufficiently large that Earth's rotation plays a significant role-in the ocean.

Given the complexity of the ocean's dynamics, which consists of interactions over a wide variety of temporal and spatial scales of motion, many important oceanographic features are quite subtle to observe and difficult to measure. Therefore, the value of having a theoretical framework that may be employed to characterize potential configurations of fluid motion, and to provide qualitative, and indeed quantitative, information on the flow, is immediately apparent (Constantin and Johnson, 2016c). There are numerous shortcomings in existing theoretical models for equatorial flows, principally due to the sheer complexity intrinsic to equatorial dynamics. Accordingly, these models do not account for a number of key oceanographic features.
In this paper we recount recent progress in modeling equatorial ocean flows by way of model equations derived directly from the fully nonlinear governing equations by a systematic asymptotic procedure (as opposed to simply satisfying ad hoc considerations based on observational data) presented in Constantin and Johnson (2017). This model generates truly nonlinear, threedimensional flows that incorporate the well-observed equatorial upwelling and downwelling phenomena as well as the Equatorial Undercurrent (EUC). (We recall both equatorial flow features in a little detail below.)

Although a simplified version of the Euler equation, derived by way of an appropriate asymptotic limiting process, these model equations remain highly complex (being nonlinear and rotational) and accordingly retain a rich mathematical and geophysical structure; these equations follow from a single overarching assumption of slow variations in the azimuthal direction in a two-layer, steady flow that is symmetric about the equator. A particularly noteworthy aspect of this system is that the explicit manifestation of Coriolis parameters in the reduced model equations enables us to determine the importance of geophysical effects in the resulting large-scale equatorial flows.

This article focuses on establishing whether nonlinear effects are also inherently important in the modeling of these equatorial phenomena. Presently, the fundamental models of oceanic circulation are based almost exclusively on linear theory, and while the conclusions derived within the linear setting are quite accurate for non-equatorial flows, it is by now well established that nonlinear effects appear at leading order in the study of equatorial ocean dynamics (see Boyd, 2018). The main thrust of this paper is to exhibit, by way of elementary mathematical exposition, that linearized flow models cannot truly capture upwelling and downwelling processes in the equatorial region. These arguments, first presented in Henry (2018), illustrate the importance of the role played by nonlinear (inertial) terms in describing equatorial ocean flow processes, and signify that an intricate understanding of nonlinear interactions is necessary in order to develop a better cognizance of large-scale ocean flows and circulation in the equatorial region. 


\section{Equatorial Ocean Flows}

The dynamics of the ocean near the equator presents some unique and complex characteristics from a modeling perspective. At the equator there is a breakdown in mid-latitude geostrophic balance, with the equator acting as a natural waveguide leading to equatorially trapped zonal waves (Fedorov and Brown, 2009; Constantin, 2012, 2013, 2014; Henry, 2016, 2017). There is pronounced stratification in the equatorial region, with a pycnocline/thermocline interface demarcating the corresponding fluid regions (Gill, 1982; Cushman-Roisin and Beckers, 2011; Constantin and Johnson, 2015, 2017; Vallis, 2017). The difference in density across the thermocline is about $1 \%$, and it is reasonable to represent the stratification as a shallow surface layer of constant density, $\rho_{0}$, overlying a deep layer of constant density, $(1+\mathrm{r}) \rho_{0}$, where $\mathrm{r} \approx 5 \times 10^{-3}$ is a typical value for this small positive constant in the equatorial Pacific (McCreary, 1985). With special regard to the model equations (and resulting equatorial ocean features) that are being considered in this paper, we outline in a little more detail two particularly fascinating oceanographic flow phenomena.

\section{UPWELLING/DOWNWELLING PROCESSES}

While the equatorial region is noted for the vanishing of the Coriolis parameter and equatorially trapped wave motion, superimposed on these flow features are regions of upwelling and downwelling (Gill, 1982; Marshall and Plumb, 2016; Vallis, 2017) that effectively convert a one-dimensional flow into a three-dimensional one. Surface upwelling is a process whereby surface water is pushed away (usually by winds blowing across the ocean surface), and is then replaced by water rising up from beneath the surface.

Upwelling occurs in the open ocean and along coastlines. The reverse process, called downwelling, typically occurs in high-latitude regions due to the formation of sea ice, because after freezing the concentration of salt in the ice is very low,

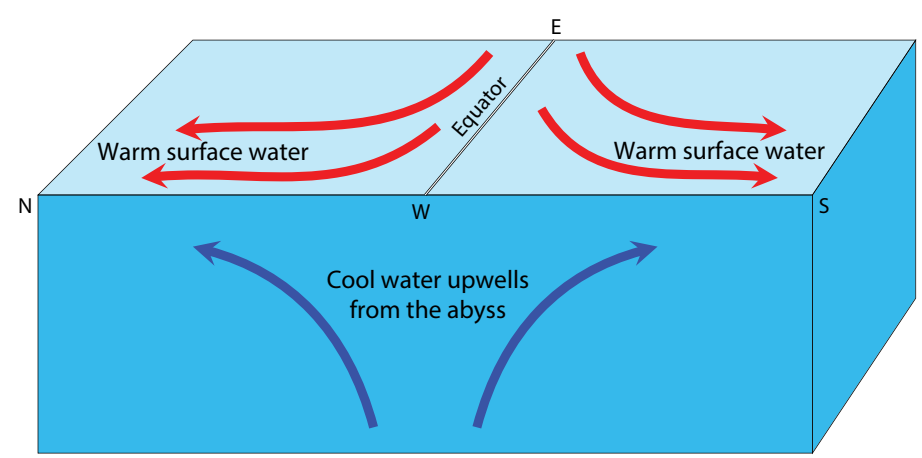

FIGURE 1. A schematic of the upwelling process, coupled with equatorial poleward flow at the surface. and hence the salt concentration in the remaining liquid water increases in a process known as brine exclusion. Actually, there are only two locations where surface waters become dense enough to sink into the deep ocean-one in the Norwegian Sea and the other in the Weddell Sea. Note that in Arctic regions, the formation of sea ice is facilitated by limited connections with the global ocean, while in the Southern Ocean, the Antarctic Circumpolar Current-the mightiest ocean current (see the discussion in Constantin and Johnson, 2016b) prevents the influence of relatively warmer subtropical ocean water (see Walton, 2013). This downwelling leads to the creation of deep-sea currents, driven by density differences but of little relevance for the near-surface equatorial ocean flow (see the discussion in Wunsch, 2015). The prevailing wind direction in the Pacific equatorial region is westward, and the surface water is usually also directed poleward due to Ekman transport effects. For the ocean in a neighborhood of the Pacific equator, this three-dimensionality results in a flow that rises to the surface all along the equator, and moves away from it close to the surface (although this upwelling may be restricted only to regions quite near the surface) as in Figure 1.

As the flow speeds are small and difficult to measure, particularly beneath the surface (flow speeds usually range from $0.1-0.2 \mathrm{~m} \mathrm{~s}^{-1}$, with maximal values at the surface reaching $1 \mathrm{~m} \mathrm{~s}^{-1}$; see Gill, 1982; Fedorov and Brown, 2009; Marshall and Plumb, 2016), it is difficult to characterize precisely the flow dynamics generated by upwelling/downwelling processes by way of direct measurements. Nevertheless, there are other means of establishing that such phenomena exist. It is known that upwelling enhances biological productivity because water that rises to the surface is typically colder and rich in nutrients. These nutrients fertilize surface waters, resulting in phytoplankton growth: it is quite clear from the satellite image in Figure 2 (where the presence of phytoplankton is manifested by a turquoise streak) that upwelling takes place in the equatorial region.

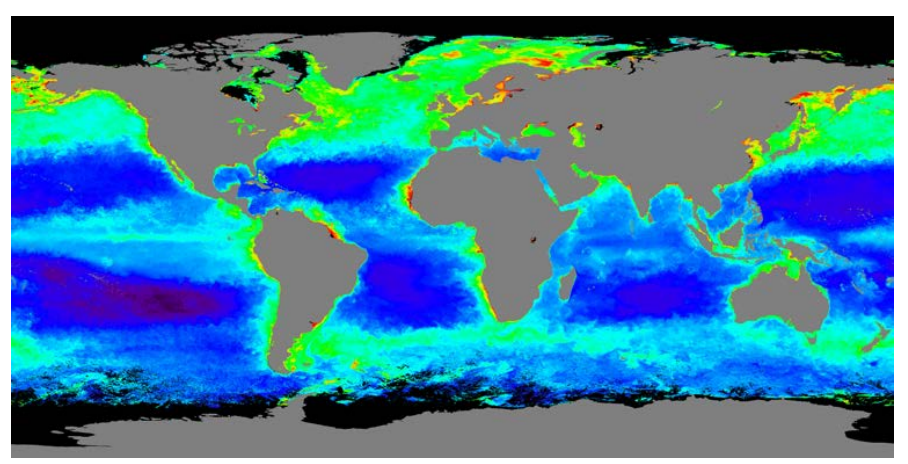

March 21-June 20, 2006

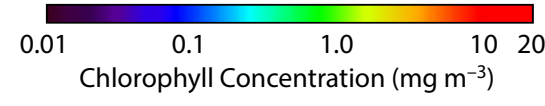

FIGURE 2. The turquoise streak along the equator shows that phytoplankton were growing along an area of equatorial upwelling. Image credit: NASA 


\section{EQUATORIAL UNDERCURRENT}

Equatorial flow dynamics are dominated by circulation that is driven by surface-wind stresses (McCreary, 1985) and the presence of non-uniform underlying currents in all three oceans. The most prominent example is probably the Pacific EUC. This is a depth-dependent current extending almost the entire length of the equatorial ocean in a band approximately $300 \mathrm{~km}$ wide about the equator. It is confined to a depth of no more than 100$200 \mathrm{~m}$ because the predominantly westward surface flow (due to prevailing winds) reverses direction at a depth of around $100 \mathrm{~m}$, resulting in a (relatively) high-speed, eastward-flowing jet whose core is aligned with the thermocline (see McCreary, 1985; Philander, 1979; Sirven, 1996; Izumo, 2005; G.C. Johnson et al., 2001; Constantin and Johnson, 2015, 2016a). Below the EUC (at depths in excess of about $240 \mathrm{~m}$ ), the motion dies out rapidly and there is an abyssal layer of essentially still water (Figure 3).

Given the massive extent of the EUC, it is amazing how elusive it remained to detection-its existence had been suspected as early as 1886, but it was not investigated properly until 1951 when it was fortuitously rediscovered by an expedition led by Townsend Cromwell (as a result, it is sometimes referred to as the "Cromwell Current"). This is even more remarkable given that the presence of the EUC, and of the westward wind-driven drift near the surface, has profound effects on equatorial ocean dynamics. The Pacific EUC is known to be especially relevant during warm episodes of the El Niño-Southern Oscillation (ENSO) phenomenon: this effect is driven by the weakened trade winds, which then allow an abnormally high eastward flow of warm water, as illustrated in Figure 4.

\section{Preliminary Considerations}

The complexity of equatorial flows from a GFD perspective is striking, and does not lend itself to optimism in the quest to derive a tractable mathematical model that captures the salient features of these flows. Therefore, it is noteworthy that significant progress has recently been achieved by Constantin and

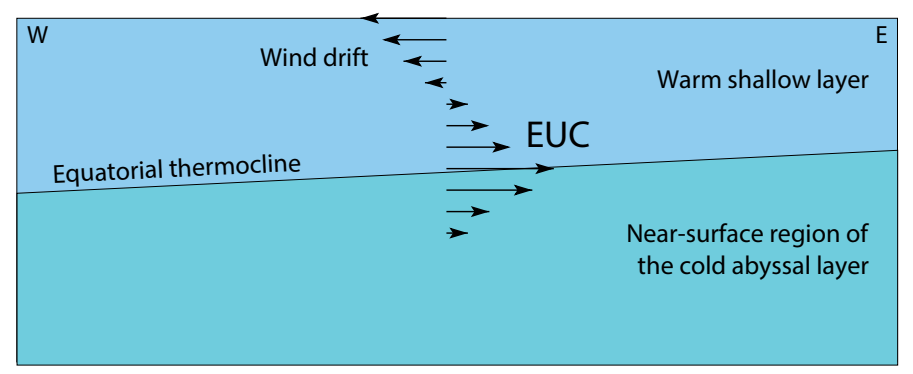

FIGURE 3. A schematic of the Equatorial Undercurrent (EUC) flow reversal.
Johnson (2017) in systematically deriving a (relatively tractable) dynamical model that is sufficiently complex to simultaneously capture oceanic phenomena such as upwelling/downwelling, depth-dependent zonal currents with flow reversal (including EUC-type flows), and poleward divergence along the equator. In this paper we describe the procedure that produces the new model equations, and outline how specific oceanographic flow processes may be described from the resulting solutions. The derivation of the dynamical model presented in Constantin and Johnson (2017) is mathematically elegant, yet it is by necessity quite technical and convoluted owing to the incorporation of both nonlinear and rotational terms. The aim of this note is to demonstrate that the retention of nonlinear effects is fundamental to the success of achieving such a rich description of the oceanic flow processes in any mathematical model. In particular, it is shown that any linearization of the model will fail to capture the emergence and persistence of large coherent structures that are representative of the equatorial ocean flows described above.

\section{EQUATIONS OF MOTION}

It is assumed that Earth is a perfect sphere of radius $\bar{R}=6,378 \mathrm{~km}$, with $\bar{\Omega}=\bar{\Omega}(0, \cos \theta, \sin \theta)$ the angular velocity vector of Earth's rotation, where $\theta$ is the angle of latitude and $\bar{\Omega}=73 \times 10^{-6} \mathrm{rad} \mathrm{s}^{-1}$ is the constant rotational speed. For oceanic flows of the type we are interested in, the Reynolds number is, in general, extremely large (Maslowe, 1986), and furthermore we may regard the fluid as incompressible (Lighthill, 1978; Constantin, 2011). The fully nonlinear, exact governing equations for geophysical fluid dynamics on a rotating sphere, assuming the fluid is inviscid and incompressible, take the form of the Euler equation

$$
\frac{D \overline{\boldsymbol{u}}}{D \bar{t}}+2 \overline{\boldsymbol{\Omega}} \times \overline{\boldsymbol{u}}=-\frac{1}{\bar{\rho}} \bar{\nabla} \bar{p}+\overline{\boldsymbol{F}},
$$

where $\overline{\boldsymbol{u}}=(\bar{u}, \bar{v}, \bar{w})$ is the fluid velocity, and fluid incompressibility is prescribed by the equation

$$
\nabla \cdot \overline{\boldsymbol{u}}=0 .
$$

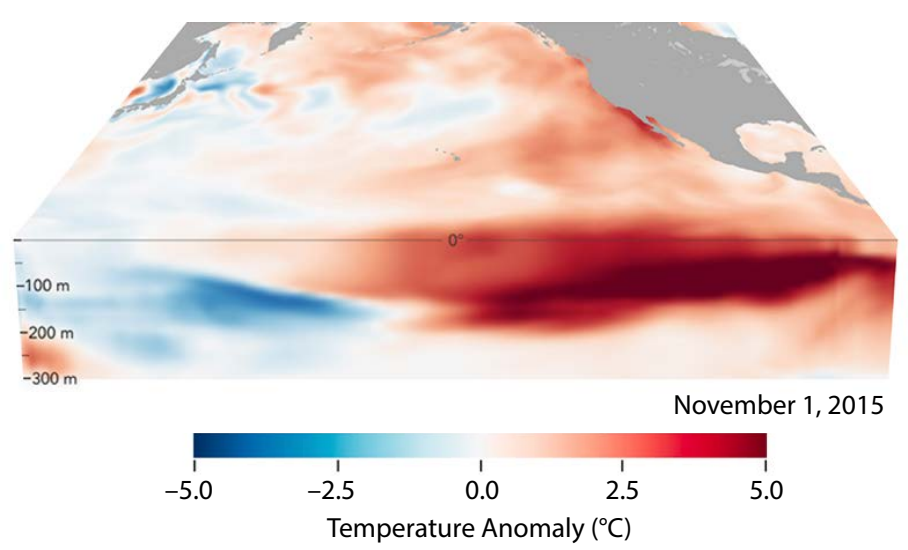

FIGURE 4. The eastward-flowing EUC transports warm water eastward beneath the surface-the warmest water is typically in the west of the Pacific Ocean. Image credit: NASA 
Here, $\overline{\boldsymbol{F}}$ is the external body force, $\bar{\rho}$ is the water density, and $\bar{p}$ is the pressure. The overbar notation employed above is motivated by various nondimensionalization and scaling procedures that we introduce below. The first term in the Euler equation (1) represents nonlinear inertial terms, while the second term is the Coriolis force, and to this point both Equations 1 and 2 are coordinate-free. In keeping with the original approach adopted in Constantin and Johnson (2017), we work with variables $\overline{\boldsymbol{x}}=(\bar{x}, \tilde{y}, \tilde{z})$ in a cylindrical coordinate system, with the origin of the coordinate system taken at a point on Earth's surface, and the $\bar{x}$-axis corresponding to the "flattened-out" great circle of the equator, which then generates the cylinder (the interior of the cylinder corresponding to the interior of the sphere). The positive $\bar{x}$-direction corresponds to azimuthal flow from west to east. In terms of cylindrical coordinates, $\tilde{y}$ is the curvilinear coordinate following the circumference of the cylinder expressed as the arc length, and $\tilde{z}$ is the radial coordinate pointing vertically upward from Earth's surface. We note that there are alternative considerations that result in the derivation of the same set of model equations (see R.S. Johnson, 2018), and this is a theme that is common in the modeling of equatorial fluid dynamics by employing mathematical approximations.

\section{Nontraditional $\beta$-Plane Equations}

In the equatorial region, whereby latitudinal variation is necessarily restricted, the governing equations are typically simplified by invoking tangent plane approximations, the classical form being the $\beta$-plane approximation (Gill, 1982; CushmanRoisin and Beckers, 2011; Vallis, 2017). The "traditional" $\beta$-plane approximation, ubiquitous in modeling equatorial flows, involves neglecting terms in the Coriolis force that feature the vertical velocity, along with the vertical component of the Coriolis force. This approximation is not canonical, in the sense that the approximation procedures employed are somewhat ad hoc and there is a wide array of research literature that proposes alternative "nontraditional" approximations for addressing various inconsistencies arising in the classical approach (see Gerkema et al., 2008; Stewart and Dellar, 2010; Constantin and Johnson, 2016a, 2017; Henry, 2016, 2017; R.S. Johnson, 2018) and the discussions therein).

For the $\beta$-plane approximation we consider, the Coriolis force terms in Equation 1 are linearized by way of approximating the terms $\sin \theta \approx \theta, \cos \theta \approx 1$ (because the latitude $\theta$ is small), leading to

$$
2 \overline{\boldsymbol{\Omega}} \times \overline{\boldsymbol{u}} \sim 2 \bar{\Omega}\left(\bar{\omega}-\frac{\tilde{y} \bar{v}}{\bar{R}}, \frac{\tilde{y} \bar{u}}{\bar{R}},-\bar{u}\right) .
$$

The associated tangent-plane Cartesian coordinate system $\{\bar{x}, \bar{y}, \bar{z}\}$ is now introduced, whereby the $\bar{x}$-axis is as before, pointing horizontally due east (zonal direction), the $\bar{y}$-axis is now pointing due north (meridional direction), and the $\bar{z}$-axis is pointing vertically upward from the tangent plane. It can be shown using elementary, yet quite intricate, geometrical considerations that the approximate identities $\tilde{y}=\bar{y}$, $\tilde{z}=\bar{z}+\bar{y}^{2} / 2 \bar{R}$, hold in the tangent plane approximation (for details, see Constantin and Johnson, 2017). The term $\bar{y}^{2} / 2 \bar{R}$ represents the drop in Earth's surface beneath the tangent plane (see Constantin and Johnson, 2017): over a distance of $10 \mathrm{~km}$ (consistent with the 0.25-degree grid size for the ocean data typically provided by NOAA) we have a departure of about $7.5 \mathrm{~m}$ from local spherical geometry on the flat-space setting inherent to the $f$-plane or $\beta$-plane approximation. Combining the above considerations, the Euler equation (1) for steady flow reduces to the following steady "nontraditional" $\beta$-plane formulation,

$$
\begin{gathered}
\bar{u} \bar{u}_{\bar{x}}+\bar{v} \bar{u}_{\bar{y}}+\bar{w} \bar{u}_{\tilde{z}}+2 \bar{\Omega}\left(\bar{w}-\frac{\bar{y}}{\bar{R}} \bar{v}\right)=-\frac{1}{\bar{\rho}_{0}} \bar{p}_{\bar{x}}, \\
\bar{u} \bar{v}_{\bar{x}}+\bar{v} \bar{v}_{\bar{y}}+\bar{w} \bar{v}_{\tilde{z}}+2 \bar{\Omega} \frac{\bar{y}}{\bar{R}} \bar{u}=-\frac{1}{\overline{\rho_{0}}} \bar{p}_{\bar{y}}, \\
\bar{u} \bar{w}_{\bar{x}}+\bar{v} \bar{w}_{\bar{y}}+\bar{w} \bar{w}_{\tilde{z}}-2 \bar{\Omega} \bar{u}=-\frac{1}{\overline{\rho_{0}}} \bar{p}_{\tilde{z}}-\bar{g},
\end{gathered}
$$

and the equation of incompressibility

$$
\bar{u}_{\bar{x}}+\bar{v}_{\bar{y}}+\bar{w}_{\tilde{z}}=0 .
$$

Here $\bar{g}=9.8 \mathrm{~m} \mathrm{~s}^{-2}$ is the constant acceleration of gravity, and $\overline{\rho_{0}}$ is the constant density of the upper fluid layer. A practically identical set of equations of motion hold in the layer beneath the thermocline except with $\overline{\rho_{0}}$ replaced by $(1+\mathrm{r}) \overline{\rho_{0}}$ : typically $\overline{\rho_{0}}=1,027 \mathrm{~kg} \mathrm{~m}^{-3}$ and $\mathrm{r}=5 \times 10^{-3}$ for the Pacific EUC. We note that Equations $4 \mathrm{a}-\mathrm{d}$ are nontraditional in the sense that they feature the curvilinear coordinate $\tilde{z}$ rather than the Cartesian version $\bar{z}$. On the free surface, denoted by $\tilde{z}=\bar{\eta}(\bar{x}, \bar{y}, \bar{t})$, we have the dynamic and kinematic boundary conditions

$$
\bar{p}=\bar{P}_{s}(\bar{x}, \bar{y}, t) \text { and } \bar{w}=\overline{u \eta}_{\bar{x}}+\overline{v \eta}_{\bar{y}}
$$

where $P_{s}$ represents the surface pressure. On the thermocline, denoted by $\tilde{z}=\bar{T}(\bar{x}, \bar{y})$, we have the kinematic boundary condition

$$
\bar{w}=\bar{u} \bar{T}_{\bar{x}}+\bar{v} \bar{T}_{\bar{y}} .
$$

Finally, on the impermeable seabed, denoted $\tilde{z}=\bar{d}(\bar{x}, \bar{y})$, we have the kinematic condition

$$
\bar{w}=\bar{u} \bar{d}_{\bar{x}}+\bar{v} \bar{d}_{\bar{y}}
$$

For flows being considered here, whereby there is no fluid motion at great depth, this last boundary condition is satisfied trivially and so Equation $5 \mathrm{c}$ can be disregarded.

\section{Model Equations}

In order to simplify the equations of motion (4) and determine the appropriate boundary conditions (5), suitable nondimensionalization and scaling procedures are invoked that employ the single overarching assumption of slow azimuthal variations. These procedures are technically intricate and quite involved, so while a brief overview is provided for completeness, we defer to Constantin and Johnson (2017) for full details. The variables 
are nondimensionalized by scaling $(\bar{x}, \bar{y}, \bar{z})=(\bar{L} x, \bar{l} y, \bar{h} z)$, $\overline{\boldsymbol{u}}=\bar{U}\left(u, \frac{\bar{l}}{\bar{L}} v, \frac{\bar{h}}{\bar{L}} \omega\right), \bar{p}=\bar{\rho}_{0} \bar{U}^{2} p$, where $(\bar{L}, \bar{l}, \bar{h})$ are length scales and $\bar{U}$ is an appropriate speed scale. Implementing the limiting procedure $(\bar{h} / \bar{L})^{2} \rightarrow 0,(\bar{l} / \bar{L})^{2}=(\bar{l} / \bar{h})^{2}(\bar{h} / \bar{L})^{2} \rightarrow 0$, reduces the equations of motion (4) to the system

$$
\begin{gathered}
u u_{x}+v u_{y}+w u_{z}+2 \omega(w-y v)=-P_{x}, \\
2 \omega y u=-P_{y}, \quad 2 \omega u=P_{z}, \\
u_{x}+v_{y}+w_{z}=0
\end{gathered}
$$

together with the boundary conditions

$$
\begin{gathered}
P(x, y, z)=P_{s}(x, y)+\frac{\bar{g} \bar{h}}{\bar{U}^{2}} \eta(x, y) \\
\text { and } w=u \eta_{x}+v \eta_{y} \text { on } z=\eta(x, y), \\
w=u T_{x}+v T_{y} \text { on } z=T(x, y) .
\end{gathered}
$$

Here $\omega=\bar{\Omega} \bar{h} / \bar{U}$ is a parameter representing the rotational Coriolis term, and $P(x, y, z)=p(x, y, z)+\frac{\bar{g} \bar{h}}{\bar{U}^{2}} z$ is the pressure relative to the hydrostatic pressure.

\section{EQUATORIAL FLOW SOLUTIONS}

We briefly present a sketch of how the system equations (6) can be solved, with complete details to be found in Constantin and Johnson (2017). Removing the pressure term from the two equations in the second line of (6) results in the equation $u_{y}+y u_{z}=0$ : this has the general solution

$$
u(x, y, z)=u\left(x, z-y^{2} / 2\right)
$$

where $u$ may be prescribed quite arbitrarily. The form of this velocity term naturally accommodates Earth's curvature relative to the tangent plane, and suggests that the free surface and thermocline have a similar structure, namely

$$
\begin{gathered}
\eta(x, y)=\frac{1}{2} y^{2}+\eta^{\prime}(x, y), \\
T(x, y)=\frac{1}{2} y^{2}-t(x) .
\end{gathered}
$$

With this formulation, and defining the new variable $\zeta=z-y^{2} / 2$, with $\phi_{\zeta}(x, \zeta)=u\left(x, z-y^{2} / 2\right)$, equations (6) and (7) have solutions of the form

$$
\begin{gathered}
v=\frac{1}{2 \omega+u_{\zeta}}\left[\left(2 \omega+u_{\zeta}\right) u u_{x \zeta}-\left(u u_{x}+2 \omega \phi_{x}\right) u_{\zeta \zeta}\right] y, \\
w=y v-\frac{u u_{x}+2 \omega \phi_{x}}{2 \omega+u_{\zeta}} .
\end{gathered}
$$

It is clear from (9) that there is some freedom in allowing the thermocline to evolve in the equatorial direction; indeed, Fedorov and Brown (2009) observed that the thermocline drops a short distance away from the western edge of the Pacific and then rises gradually toward the east at a rate of about $1 \mathrm{~m}$ over one degree of longitude at the equator, and this behavior can be easily captured by (9). Furthermore, the formulation of (9) permits variability in the free surface in both the azimuthal and latitudinal directions, enabling the modeling of surface distortion in the equatorial region. This is relevant in the Pacific EUC context as it is a property of the Pacific that the level of the ocean rises by about $0.5 \mathrm{~m}$ from east to west relative to Earth's curvature because of the action of the trade winds.

With regard to the velocity field in (8), (10), and (11), it is apparent that the flow structure is truly nonlinear and threedimensional, and furthermore the influence of the Coriolis terms is readily apparent from the $\omega$ parameter. Furthermore, the azimuthal velocity in (8) can be prescribed with a large degree of freedom, and it is possible to use (8) to model a depth-varying current exhibiting flow reversal as evidenced by the EUC; following the prescription of $u$, a three-dimensional flow is generated by way of (10) and (11). Indeed, Constantin and Johnson (2017) explicitly demonstrate through examples that the flow determined by the above solution (8), (10), and (11) can be prescribed to model EUC-type flows interacting with upwelling and downwelling processes, thereby capturing elements of the equatorial ocean flow that have hitherto evaded modeling by way of equations systematically derived from the full governing equations.

\section{Linear Flow Solutions}

Having described a nonlinear three-dimensional flow solution above that models upwelling and downwelling, we examine whether such equatorial ocean flows are in the remit of linearized fluid motions. Let us assume that the fluid velocity field is three-dimensional, and that is has a linear representation: this implies that $\dot{X}=A X$, where $A$ is a matrix and $\boldsymbol{X}(t)=(X(t), Y(t), Z(t))$ represent the variables in any appropriate coordinate system. An extensive mathematical discussion of (two-dimensional) linear fluid motion can be found in Constantin (2011), and some relatively intricate linear flow patterns are presented and discussed in Majda and Bertozzi (2002). Accordingly, the three-dimensional fluid motion can be expressed component-wise as

$$
\begin{aligned}
U & =a_{11} X+a_{12} Y+a_{13} Z, \\
V & =a_{21} X+a_{22} Y+a_{23} Z, \\
W & =a_{31} X+a_{32} Y+a_{33} Z .
\end{aligned}
$$

This system (12) has the fundamental solution (see Meiss, 2007) given by $\boldsymbol{X}(t)=\boldsymbol{X}_{0} e^{A t}$, where $\boldsymbol{X}_{0}$ is the initial position of the fluid parcel. As equatorial fluid motion is characteristically symmetric about the equator, we assume that $(X(t), Y(t), Z(t))=(X(t),-Y(t), Z(t))$, that is, $\left(X_{0}, Y_{0}, Z_{0}\right) e^{A t}=$ $\left(X_{0},-Y_{0}, Z_{0}\right) e^{A t}$. Differentiating this expression with respect to $t$, and setting $t=0$, we deduce that $A$ has the eigenvector $e_{0}=(0,1,0)$ corresponding to the eigenvalue 0 . From the conservation of mass, prescribed by Equation $4 \mathrm{~d}$, we infer that $a_{11}+a_{22}+a_{33}=0$, that is, $\operatorname{tr} A=0$. Piecing this information together, we conclude that the characteristic equation for $A$ is $\lambda^{3}+q \lambda=0$. Since the coefficients of $A$ are real, the constant $q$ must be real, and accordingly we must consider three distinct scenarios. 


\section{CASE 1}

If $q=0$, then the Jordan canonical form for $A$ is either 0 , or takes the form

$$
\left(\begin{array}{lll}
0 & 1 & 0 \\
0 & 0 & 1 \\
0 & 0 & 0
\end{array}\right)
$$

In this case, we have

$$
e^{A t}=\left(\begin{array}{ccc}
1 & t & \frac{1}{2} t^{2} \\
0 & 1 & t \\
0 & 0 & 1
\end{array}\right)
$$

Therefore, $X(t)=\left(X_{0}+Y_{0} t+\frac{Z_{0}}{2} t^{2}, Y_{0}+Z_{0} t, Z_{0}\right)$, and consequently $\boldsymbol{X}^{\prime \prime}(t)=(c, 0,0)$. This implies a uniform zonal acceleration for each fluid particle, which is not plausible in the context of equatorial ocean flows.

\section{CASE 2}

If $q<0$, then the eigenvalues are $\lambda=0, \pm p$, where $p^{2}=-q$ for $p>0$. This scenario implies the existence of both a stable and unstable manifold, which does not match the steady flow exhibited in Constantin and Johnson (2017), where the flow is toward the west and poleward in the near-surface layer.

\section{CASE 3}

Supposing that $q>0$ leads to the eigenvalues $\lambda=0, \pm i p$, where $p>0$ is such that $p^{2}=q$. In this case, all particle paths would be periodic, which does not capture the equatorial flow we are considering.

In conclusion, we have shown that any inherently linear fluid motion is limited to modeling a constrained range of equatorial ocean flows. Specifically, linear fluid flows cannot capture the upwelling effect whereby close to the surface the equatorial ocean flow moves westward along the equator while simultaneously moving away from the equator poleward. @

\section{REFERENCES}

Boyd, J.P. 2018. Dynamics of the Equatorial Ocean. Springer, Berlin.

Constantin, A. 2011. Nonlinear Water Waves with Applications to Wave-Current Interactions and Tsunamis. Vol. 81 in CBMS-NSF Conference Series in Applied Mathematics, Society for Industrial and Applied Mathematics, https://doi.org/ 10.1137/1.9781611971873.

Constantin, A. 2012. An exact solution for equatorially trapped waves. Journal of Geophysical Research 117, C05029, https://doi.org/10.1029/2012JC007879.

Constantin, A. 2013. Some three-dimensional nonlinear equatorial flows. Journal of Physical Oceanography 43:165-175, https://doi.org/10.1175/JPO-D-12-062.1.

Constantin, A. 2014. Some nonlinear, equatorially trapped, nonhydrostatic internal geophysical waves. Journal of Physical Oceanography 44:781-789, https://doi.org/10.1175/JPO-D-13-0174.1.

Constantin, A., and R.S. Johnson. 2015. The dynamics of waves interacting with the Equatorial Undercurrent. Geophysical \& Astrophysical Fluid Dynamics 109:311-358, https://doi.org/10.1080/03091929.2015.1066785.

Constantin, A., and R.S. Johnson. 2016a. An exact, steady, purely azimuthal equatorial flow with a free surface. Journal of Physical Oceanography 46:1,935-1,945, https://doi.org/10.1175/JPO-D-15-0205.1.

Constantin, A., and R.S. Johnson. 2016b. An exact, steady, purely azimuthal flow as a model for the Antarctic Circumpolar Current. Journal of Physical Oceanography 46:3,585-3,594, https://doi.org/10.1175/JPO-D-16-0121.1.

Constantin, A., and R.S. Johnson. 2016c. Current and future prospects for the application of systematic theoretical methods to the study of problems in physical oceanography. Physics Letters A 380:3,007-3,012, https://doi.org/10.1016/ j.physleta.2016.07.036.
Constantin, A., and R.S. Johnson. 2017. A nonlinear, three-dimensional model for ocean flows, motivated by some observations of the Pacific equatorial undercurrent and thermocline. Physics of Fluids 29, 056604, https://doi.org/ 10.1063/1.4984001.

Cushman-Roisin, B., and J.-M. Beckers. 2011. Introduction to Geophysical Fluid Dynamics: Physical and Numerical Aspects. Academic Press, Waltham, MA, 875 pp.

Fedorov, A.V., and J.N. Brown. 2009. Equatorial waves. Pp. 3,679-3,695 in Encyclopedia of Ocean Sciences, J. Steele, ed., Academic Press, San Diego.

Gerkema, T., J.T.F. Zimmerman, L.R.M. Maas, and H. van Haren. 2008. Geophysical and astrophysical fluid dynamics beyond the traditional approximation. Reviews of Geophysics 46, RG2004, https://doi.org/10.1029/2006RG000220.

Gill, A. 1982. Atmosphere-Ocean Dynamics. New York: Academic Press, 662 pp.

Henry, D. 2016. Equatorially trapped nonlinear water waves in a $\beta$-plane approximation with centripetal forces. Journal of Fluid Mechanics 804, R1, https://doi.org/ 10.1017/jfm.2016.544.

Henry, D. 2017. A modified equatorial $\beta$-plane approximation modelling nonlinear wave-current interactions. Journal of Differential Equations 263:2,554-2,566, https://doi.org/10.1016/j.jde.2017.04.007.

Henry, D. 2018. On nonlinearity in three-dimensional equatorial flows. Journal of Nonlinear Mathematical Physics 25:351-357, https://doi.org/10.1080/14029251. 2018.1494780.

Izumo, T. 2005. The equatorial current, meridional overturning circulation, and their roles in mass and heat exchanges during the El Niño events in the tropical Pacific Ocean. Ocean Dynamics 55:110-123, https://doi.org/10.1007/ s10236-005-0115-1.

Johnson, G.C., M.J. McPhaden, and E. Firing. 2001. Equatorial Pacific Ocean horizontal velocity, divergence, and upwelling. Journal of Physical Oceanography 31(3):839-849, https://doi.org/10.1175/1520-0485(2001)031 $<0839$ :EPOHVD>2.0.CO;2.

Johnson, R.S. 2018. Application of the ideas and techniques of classical fluid mechanics to some problems in physical oceanography. Philosophical Transactions of the Royal Society A 276, 20170092, https://doi.org/10.1098/ rsta.2017.0092.

Lighthill, J. 1978. Waves in Fluids. Cambridge University Press, Cambridge and New York.

Majda, A.J., and A.L. Bertozzi. 2002. Vorticity and Incompressible Flow. Cambridge University Press, Cambridge.

Marshall, J., and R.A. Plumb. 2016. Atmosphere, Ocean and Climate Dynamics: An Introductory Text. Academic Press, London.

Maslowe, S.A. 1986. Critical layers in shear flows. Annual Review of Fluid Mechanics 18:405-432, https://doi.org/10.1146/annurev.fl.18.010186.002201.

McCreary, J.P. 1985. Modeling equatorial ocean circulation. Annual Review of Fluid Mechanics 17(1):359-409, https://doi.org/10.1146/annurev.fl.17.010185.002043.

Meiss, J.D. 2007. Differential Dynamical Systems. Society for Industrial and Applied Mathematics, Philadelphia, https://doi.org/10.1137/1.9780898718232.

Philander, S.G.H. 1979. Equatorial waves in the presence of the equatorial undercurrent. Journal of Physical Oceanography 9:254-262, https://doi.org/10.1175/ 1520-0485(1979)009<0254:EWITPO>2.0.CO;2.

Sirven, J. 1996. The equatorial undercurrent in a two layer shallow water model. Journal of Marine Systems 9:171-186, https://doi.org/10.1016/ S0924-7963(96)00041-3.

Stewart, A.L., and P.J. Dellar. 2010. Multilayer shallow water equations with complete Coriolis force: Part I. Derivation on a non-traditional beta-plane. Journal of Fluid Mechanics 651:387-413, https://doi.org/10.1017/S0022112009993922.

Vallis, G.K. 2017. Atmospheric and Oceanic Fluid Dynamics, 2nd ed. Cambridge University Press.

Walton, D.W.H. 2013. Antarctica: Global Science from a Frozen Continent. Cambridge University Press, Cambridge, 352 pp.

Wunsch, C. 2015. Modern Observational Physical Oceanography. Princeton University Press, New Jersey, 512 pp.

\section{ACKNOWLEDGMENTS}

This research was supported by Science Foundation Ireland (SFI) grant 13/CDA/2117. The author acknowledges the support received during his stay at the ESI, Vienna, during the program "Mathematical Aspects of Physical Oceanography" in 2018.

\section{AUTHOR}

David Henry (d.henry@ucc.ie) is a lecturer in applied mathematics at University College Cork, Ireland.

\section{ARTICLE CITATION}

Henry, D. 2018. Nonlinear features of equatorial ocean flows. Oceanography 31(3):22-27, https://doi.org/10.5670/oceanog.2018.305. 